\title{
Bilateral pulmonary emboli in a teenager with positive SARS-CoV-2 antibody
}

\author{
John Kotula ${ }^{1}$, Niveditha Balakumar ${ }^{1}$, Danyal Khan ${ }^{1}$, and Bhavi Patel ${ }^{1}$ \\ ${ }^{1}$ Nicklaus Children's Hospital
}

July 28,2020

\begin{abstract}
Thromboembolic phenomena, especially pulmonary emboli, have been described in adult patients with COVID, but have been less evident in children. We describe a case of a teenager with bilateral pulmonary emboli leading to cardiovascular collapse in the setting of COVID and multisystem inflammatory syndrome in children (MIS-C).
\end{abstract}

\section{Introduction}

SARS-CoV-2 has been associated with an increased incidence of thromboembolic events, including pulmonary embolism, in up to $20 \%$ of adult patients with COVID-19 ${ }^{1}$. While the data in pediatric patients are less robust, older teenagers with MIS-C have been reported to have pulmonary embolism ${ }^{2}$. MIS-C has been defined in recent literature by the six following criteria: serious illness leading to hospitalization, age less than 21 years, fever $>38.0^{\circ} \mathrm{C}$ or report of subjective fever lasting at least 24 hours, laboratory evidence of inflammation, multisystem organ involvement involving at least two systems, and laboratory confirmed SARS-CoV-2 infection (positive SARS-CoV-2 PCR or positive antibody test during hospitalization) or an epidemiologic link to a person with COVID- $19^{2}$. We describe the case of a 15 year old female with a positive SARS-CoV-2 IgM antibody test during admission with bilateral pulmonary emboli requiring catheter directed thrombolysis for resolution.

\section{Case Report}

A 15-year old obese female with a measured weight of 105 kilograms (BMI $34.29 \mathrm{~kg} / \mathrm{m}^{2}$ ) with a past medical history of asthma who underwent a laparoscopic appendectomy 3 days prior to admission presented to an emergency department at an outside hospital with shortness of breath and 2 episodes of syncope. She had a respiratory rate of 50 breaths per minute with oxygen saturations of $100 \%$ on non-rebreather mask along with hypertension to 150/111 millimeters of mercury ( $\mathrm{mmHg}$ ). She suddenly became bradycardic followed by multiple asystolic cardiac arrests with brief return of systemic circulation (ROSC) lasting a total of 40 minutes requiring chest compressions and multiple epinephrine doses. After ROSC, she was intubated for cardiorespiratory failure, started on norepinephrine for hypotension, and given systemic tissue plasminogen activator (tPA) for concerns of pulmonary embolism (PE). Computed tomography angiography (CTA) of the chest showed multiple bilateral pulmonary emboli in the secondary and tertiary branches of the pulmonary arteries and potential thrombus in the superior vena cava. CT of the abdomen showed post-operative changes from the recent appendectomy and CT of the brain was normal. Post arrest, she had a lactic acidosis to 17 millimoles per liter with a $\mathrm{pH}$ of 6.819 . Her serum laboratory values were also significant for leukocytosis, hyperglycemia and hypoalbuminemia as well as elevated troponin-I, erythrocyte sedimentation rate (ESR), C-reactive protein (CRP), alanine transaminase (ALT) and aspartate transaminase (AST), D-dimer, blood urea nitrogen $(\mathrm{BUN})$, and creatinine $(\mathrm{Cr})$ (Table 1). She was transferred to a tertiary care facility for further management. 
Upon arrival, she was noted to be febrile and had supraventricular tachycardia (SVT) at 220 beats per minute (bpm) which converted to sinus rhythm after 2 doses of adenosine. Blood pressures were maintained on an epinephrine infusion. Echocardiogram showed moderately depressed right ventricular function. She was emergently taken for cardiac catheterization which showed severely elevated right atrial pressures of 20mmHg. Right ventricular pressure was a systolic of $32 \mathrm{mmHg}$ with a severely elevated end diastolic pressure of $24 \mathrm{mmHg}$. Patient had low cardiac output with a measured cardiac Index of 2.6 liters per minute per meter squared. Bilateral uni-fuse catheters were placed in the left and right pulmonary arteries for localized TPA infusion. She received 24 hours of localized TPA therapy at 0.5 milligrams per hour via each catheter along with heparin infusion (Figure 1). Lactic acid level and right ventricle function normalized on the echocardiogram 2 days after admission, and CTA of the chest showed resolution of bilateral PE on day 3 of admission. She was successfully extubated after 12 days and required hemodialysis for kidney failure for 7 days. She had multiple episodes of fevers throughout her admission, and further infectious workup showed SAR-CoV2-IgM to be positive. SAR-CoV2 PCR was negative on a total of six tests during admission, and the SAR-CoV2-IgG remained negative on a total of three tests. There was no deep venous thrombosis found on ultrasonography, and the hypercoagulable workup only showed elevated homocysteine levels likely due to kidney failure. She suffered from neurological devastation with spasticity from hypoxic ischemic injury noted on magnetic resonance imaging of the brain in the occipital, parietal, frontal gray-white matter bilaterally. Gastrostomy tube was placed for feeding. She was discharged to an inpatient neurological rehabilitation center.

\section{Discussion}

This case highlights the relationship between likely SARS-CoV-2 associated MIS-C and thrombotic complications, particularly pulmonary embolism. Although this patient's SARS-CoV-2 PCR was negative multiple times, the SARS-CoV-2 IgM antibody was positive during hospitalization. Her positive antibody test coupled with fever, age less than 21 years, hospitalization, laboratory evidence of inflammation and evidence of multisystem involvement fit reported criteria for MIS-C ${ }^{2}$. The negative PCR is not unusual in MIS-C cases; recent evidence has demonstrated only $70 \%$ of patients have PCR or antibody evidence of SARS-CoV-2 infection and up to $44 \%$ of reported pediatric patients with MIS-C have a negative or unknown SARS-CoV-2 PCR test during hospitalization ${ }^{2}$.

Thrombotic complications have been described in adults in both previous case reports and more recently in large case series and prospective studies ${ }^{1,3,4}$. A case series in a French hospital reported rates of pulmonary embolism of $20.6 \%$ of COVID-19 patients, rates twice as high compared to a similar time period in 2019 in their institution. A multicenter study also in France reported significantly higher rates of pulmonary embolism of $16.7 \%$ in COVID-19 ARDS patients vs. $1.3 \%$ in non-COVID-19 ARDS patients, and this association persisted after matching

between groups ${ }^{4}$. The high incidence of thrombotic complications amongst COVID-19 has led to much discussion regarding appropriate prevention of deep vein thrombosis, with pharmacologic VTE prophylaxis recommended in hospitalized patients without other risk factors ${ }^{5,6}$.

Thrombotic complications in children with COVID-19 are not as well described as in adults, perhaps due to the less severe nature of the disease in younger patients. To our knowledge, the youngest patient described in a case report prior to this case is a 17 year old pregnant female ${ }^{7}$. Thrombotic complications with MIS-C are relatively uncommon, but increase with age, with the incidence highest in the 13-20 year age group at a rate of $7 \%^{2}$. The patient in this case had a particular set of known risk factors that likely contributed to her thrombotic complications, particularly obesity and recent surgery.

Given the increased incidence of pulmonary embolism in COVID-19 in older teenagers, pediatric providers taking care of these patients may be confronted with treatment decisions regarding pulmonary embolism. In this patient, catheter directed thrombolysis for twenty-four hours provided resolution of bilateral pulmonary emboli on CT angiography within three days. Catheter directed thrombolysis has previously been described as safe and effective in pediatric patients, and has recently been described in a case report for an adult 
patient with COVID-19 and pulmonary embolus ${ }^{8,9}$. In pediatric patients with massive pulmonary embolus and COVID-19, it should also be considered as definitive therapy. Despite the successful treatment of the pulmonary emboli in this patient, unfortunately, due to her associated cardiac arrest, her overall neurologic outcome remains poor.

\section{References}

1. Poissy J, Goutay J, Caplan M, Parmentier E, Duburcq T, Lassalle F, Jeanpierre E, Rauch A, Labreuche J, Susen S. Pulmonary Embolism in COVID-19 Patients: Awareness of an Increased Prevalence. Circulation. 2020:184-186. doi:10.1161/CIRCULATIONAHA.120.047430

2. Feldstein LR, Rose EB, Horwitz SM, Collins JP, Newhams MM, Son MBF, Newburger JW, Kleinman LC, Heidemann SM, Martin AA, et al. Multisystem Inflammatory Syndrome in U.S. Children and Adolescents. The New England journal of medicine. 2020. http://www.ncbi.nlm.nih.gov/pubmed/32598831. doi:10.1056/NEJMoa2021680

3. Ullah W, Saeed R, Sarwar U, Patel R, Fischman DL. COVID-19 Complicated by Acute Pulmonary Embolism and Right-Sided Heart Failure. JACC: Case Reports. 2020;2(9):1379-1382. https://doi.org/10.1016/j.jaccas.2020.04.008. doi:10.1016/j.jaccas.2020.04.008

4. Helms J, Tacquard C, Severac F, Leonard-Lorant I, Ohana M, Delabranche X, Merdji H, ClereJehl R, Schenck M, Fagot Gandet F, et al. High risk of thrombosis in patients with severe SARSCoV-2 infection: a multicenter prospective cohort study. Intensive Care Medicine. 2020;46(6):1089-1098. https://doi.org/10.1007/s00134-020-06062-x. doi:10.1007/s00134-020-06062-x

5. Thachil J, Tang N, Gando S, Falanga A, Cattaneo M, Levi M, Clark C, Iba T. ISTH interim guidance on recognition and management of coagulopathy in COVID-19. Journal of Thrombosis and Haemostasis. 2020;(March):1023-1026. doi:10.1111/jth.14810

6. Barnes GD, Burnett A, Allen A, Blumenstein M, Clark NP, Cuker A, Dager WE, Deitelzweig SB, Ellsworth S, Garcia D, et al. Thromboembolism and anticoagulant therapy during the COVID-19 pandemic: interim clinical guidance from the anticoagulation forum. Journal of Thrombosis and Thrombolysis. 2020;50(1):7281. https://doi.org/10.1007/s11239-020-02138-z. doi:10.1007/s11239-020-02138-z

7. Martinelli I, Ferrazzi E, Ciavarella A, Erra R, Iurlaro E, Ossola M, Lombardi A, Blasi F, Mosca F, Peyvandi F. Pulmonary embolism in a young pregnant woman with COVID-19. Thrombosis Research. 2020;191(April):36-37. https://doi.org/10.1016/j.thromres.2020.04.022. doi:10.1016/j.thromres.2020.04.022

8. Bavare AC, Naik SX, Lin PH, Poi MJ, Yee DL, Bronicki RA, Philip JX, Desai MS. Catheterdirected thrombolysis for severe pulmonary embolism in pediatric patients. Annals of Vascular Surgery. 2014;28(7):1794.e1-1794.e7. http://dx.doi.org/10.1016/j.avsg.2014.03.016. doi:10.1016/j.avsg.2014.03.016

9. Qanadli SD, Gudmundsson L, Rotzinger DC. Catheter-directed thrombolysis in COVID-19 pneumonia with acute PE: Thinking beyond the guidelines. Thrombosis Research. 2020;192(April):9-11. doi:10.1016/j.thromres.2020.05.007

\section{Disclosure and Freedom of Investigation}

There was no funding used to support this project. The authors had full control of the production of the written report. The authors do not have any conflict of interests.

\section{Hosted file}

Table 1 for COVID PE case.docx available at https://authorea.com/users/346946/articles/ 472740-bilateral-pulmonary-emboli-in-a-teenager-with-positive-sars-cov-2-antibody

\section{Hosted file}


Figure 1 COVID PE case.eps available at https://authorea.com/users/346946/articles/472740bilateral-pulmonary-emboli-in-a-teenager-with-positive-sars-cov-2-antibody 